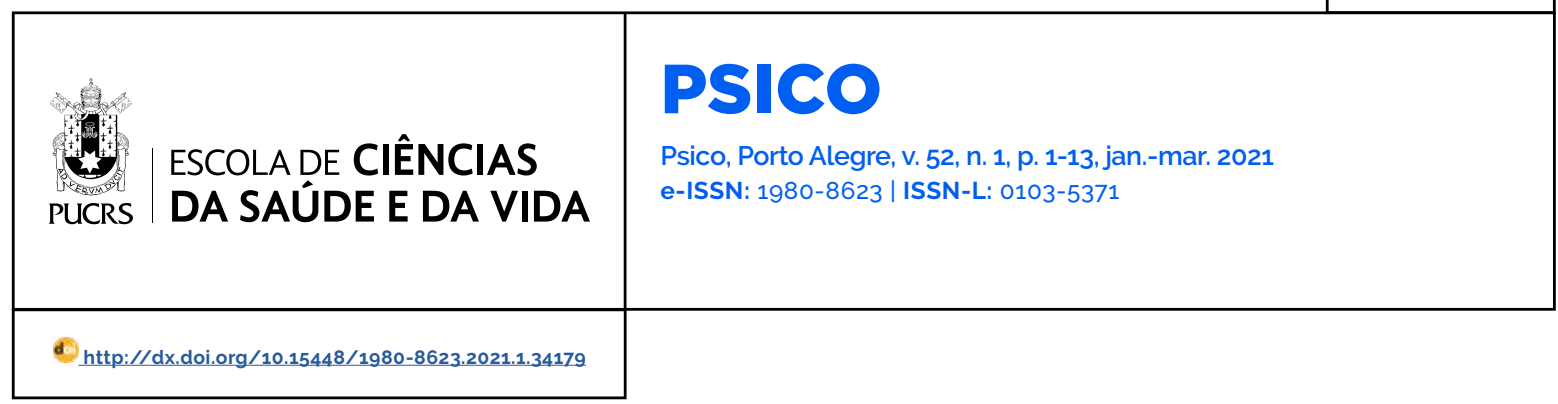

SEÇÃO: ARTIGO

\title{
Otimismo e ganho percebido em cuidadores de crianças com câncer
}

\author{
Optimism and benefit finding in caregivers of children with cancer \\ Optimismo y crecimiento psicológico postraumático en los cuidadores de niños con câncer
}

\author{
Larissa Bessert Pagung ${ }^{1}$ \\ orcid.org/0000-0002-5106-6267 \\ larissapagung@gmail.com
}

Kelly Ambrósio Silveira ${ }^{1}$ orcid.org/0000-0002-2381-2322 kellyasfs@gmail.com

\section{Alessandra Brunoro \\ Motta $^{1}$}

orcid.org/0000-0003-1162-185X

alessandrabmotta@yahoo.com.br

Recebido em: 15 maio 2019. Aprovado em: 14 out. 2020 Publicado em: 17 jun. 2021

\section{(c) (1)}

Artigo está licenciado sob forma de uma licença Creative Commons Atribuição 4.0 Internacional.
Resumo: Com o objetivo de analisar as relações entre otimismo e ganho percebido em cuidadores de crianças com câncer, constituiu-se uma amostra de conveniência de 60 cuidadores, com média de idade de 36,5 anos ( $D P=9,17$ ), $81,7 \%$ de mulheres, em um hospital de referência. Utilizaram-se os instrumentos: Teste de Orientação da Vida, Inventário de Desenvolvimento Pós-Traumático, e questionário sociodemográfico e clínico. Após análise estatística descritiva e inferencial, verificou-se correlação positiva entre otimismo e ganho percebido (percepção de recursos e competências pessoais). Otimismo e ganho percebido também se associaram às variáveis clinicas e sociodemográficas: cuidadores casados e com crianças fora de quimioterapia referiram maior ganho percebido: e cuidadores cujos filhos tinham mais tempo de tratamento, se mostraram mais otimistas e com maior ganho percebido. Conclui-se que caracteristicas individuais e clínicas devem ser consideradas em intervenções com foco na ressignificação da experiência de ter um filho com câncer e o crescimento frente à adversidade. Palavras-chave: cuidadores, otimismo, crescimento psicológico pós-traumático, criança, oncologia

Abstract: In order to analyze the relationship between optimism and benefit finding in caregivers of children with cancer, a convenience sample with 60 caregivers was made up, with an average age of 36.5 (DP=9.17), 81.7 women, in a referral hospital. The tools used were: Life Orientation Test, Post-traumatic Growth Inventory and socio-demographic and clinical questionnaire. After the descriptive and inferential statistical analysis, the positive correlation between optimism and benefit finding (perception of resources and personal skills) was observed. Optimism and benefit finding were also associated with clinical and socio-demographic variables: married caregivers and with children without chemotherapy reported greater benefit finding; and caregivers whose children had a longer treatment period turned out to be more optimistic and with greater benefit finding. It is concluded that individual and clinical characteristics should be considered in interventions focused on reframing the experience of having a child with cancer and the growth face to adversities.

Keywords: caregivers, optimism, post-traumatic growth, children, oncology

Resumen: Para analizar la relación entre optimismo y crecimiento psicológico postraumático en los cuidadores de niños con cáncer, se constituyó una muestra de conveniencia de 60 cuidadores, con una edad media de 36,5 años (DE = 9,17), $81,7 \%$ de mujeres, en un hospital de referencia. Los instrumentos utilizados fueron: Life Orientation Test, Posttraumatic Growth Inventory y cuestionario sociodemográfico y clínico. Tras el análisis estadistico descriptivo e inferencial, se constató una correlación positiva entre el optimismo y la ganancia percibida (Percepción de recursos y habilidades personales). El optimismo y la ganancia percibida también se asociaron con variables clínicas y sociodemográficas: los cuidadores casados y los niños sin quimioterapia reportaron una mayor ganancia percibida; y los cuidadores cuyos hijos tuvieron más tiempo de tratamiento, fueron 
más optimistas y percibieron una mayor ganancia. Se concluye que las caracteristicas individuales y clínicas deben ser consideradas en intervenciones enfocadas a replantear la experiencia de tener un hijo con cáncer y crecimiento ante la adversidad.

Palabras clave: cuidadores, optimismo, crecimiento psicológico postraumático, niño, oncología

O câncer é uma doença que vem acometendo mais vítimas a cada ano no mundo inteiro, atingindo, inclusive, crianças. O Instituto Nacional de Câncer José Alencar Gomes da Silva (INCA) estima que para o biênio de 2020-2022, a estimativa é de que surjam 625 mil novos casos de câncer no Brasil, para cada ano (Ministério da Saúde, 2020). Nesse contexto, pesquisadores na área da Psicologia da Saúde, vêm percebendo necessidade de mais pesquisas, tanto com crianças portadoras de câncer, como também com seus cuidadores e familiares, buscando assim uma melhor compreensão deste momento (Compas et al., 2014; Willard et al., 2016; Gardner et al., 2017; Wiener et al., 2015).

Os estudos com pais e cuidadores de crianças com câncer têm enfocado, principalmente, no sofrimento psicológico vivenciado pelos cuidadores (de Siqueira et al., 2019), no impacto profissional e financeiro associado ao tratamento oncológico da criança (da Silva et al., 2019), nas mudanças nas práticas educativas, nas alterações na dinâmica familiar e na influência de práticas culturais na vivência do tratamento (Kohlsdorf \& Costa Júnior, 2012). Essas variáveis relacionadas à familia compõem um conjunto de caracteristicas psicossociais que precisam ser compreendidas pela equipe de saúde, de modo que a assistência à criança em tratamento atenda às demandas de alta complexidade do câncer, tanto no que se refere aos aspectos biomédicos, como também aos aspectos psicossociais (Kazak \& Baxt, 2007).

Juntamente com a criança, os pais sofrem os efeitos da doença e do tratamento, os quais incluem reações emocionais variadas e mudanças nas relações familiares, que abrangem a alternância dos papéis de cada membro no funcionamento da familia. Em busca de ajustamento, a familia precisa encontrar formas de manter uma rotina dinâmica, incorporando os aspectos mais desafiadores que se colocam, como os tratamentos e as mudanças no contexto social e familiar (Hildenbrand et al., 2014).

Devido aos desafios de um tratamento oncológico infantil, os pais ou cuidadores acabam por ter um papel mais ativo no cuidado à criança. Esses cuidados se estendem desde aqueles mais simples em casa, até o acompanhamento em consultas e tratamentos - como quimioterapia. $\mathrm{O}$ ato de cuidar de uma criança com câncer impõe demandas diferentes e desafiadoras que podem comprometer a saúde física e emocional do cuidador (Salvador et al., 2015).

Pensando na saúde emocional desses cuidadores, autores como Fotiadou et al. (2008) afirmam que algumas características pessoais, como o otimismo, podem favorecer o enfrentamento ao adoecimento do filho. O otimismo parece ser um recurso pessoal importante para os pais de crianças com câncer, o que tem gerado interesse nos pesquisadores (Bozkurt et al., 2019). Entretanto, estudos sobre o otimismo no contexto de cuidadores de crianças com doença crônica têm recebido pouca atenção.

O otimismo, no campo das emoções positivas, assim como o bem-estar subjetivo, a alegria e a autodeterminação, tem se tornado componente teórico fundamental. Seligman e Csikszentmihalyias (2014) referem que nas últimas três décadas o otimismo tem sido extensamente estudado, resultando em inúmeras produções científicas com achados impressionantes. De acordo com Segerstrom, Carver e Scheier (2017), pessoas otimistas tendem a ser mais confiantes e persistentes, e o otimismo pode ser preditor e promotor de bem-estar, especialmente em situações desafiantes ou estressantes.

Pensando em formas de medir o otimismo, Scheier e Carver (1985) desenvolveram o Life Orientation Test (Teste de Orientação para a Vida), passando a estudar o otimismo em diferentes grupos. Os autores definem o otimismo como uma disposição geral em esperar bons resultados, especificamente como forma de orientação para a vida, sendo mais otimista ou mais pessimista. 
Uma orientação otimista de vida está também relacionada à promoção de resiliência psicológica e resultados positivos de saúde em portadores de doenças crônicas (Williams et al., 2010).

Outro construto que tem sido estudado no campo da saúde, é o ganho percebido. O ganho percebido envolve o processo de reavaliar circunstâncias adversas de forma positiva, diminuindo as implicações negativas do processo, bem como protegendo a autoestima (Taylor, 1983). E, em outras palavras, como afirmam Seligman e Csikszentmihalyi (2014), o ganho percebido se refere a encontrar benefícios na adversidade, ideia que vem de encontro com o surgimento da Psicologia Positiva.

Phipps (2007) buscou relacionar otimismo e ganho percebido em sua pesquisa com crianças com câncer. Os resultados apontaram uma relação positiva entre estes dois construtos, indicando que pessoas otimistas conseguem perceber ganhos após um evento traumático - como o câncer, por exemplo. Em estudo com adultos sobreviventes do câncer infantil, Yi et al. (2015) descobriram que o otimismo estava correlacionado positivamente tanto com o crescimento pós-traumático, quanto com melhor qualidade de vida na vida adulta. Com os cuidadores de crianças sobreviventes com câncer, os achados vão na mesma direção, indicando que encontrar benefícios na adversidade pode ter um impacto positivo, em termos de qualidade de vida, para esses cuidadores (Gardner et al., 2017).

Uma variedade de tipos de ganho, em relação à experiência de uma doença, tem sido relatada pela literatura, por pessoas com doenças crônicas diferentes (Harding et al., 2014; Mavandadi et al., 2014), como relacionamentos fortalecidos, crescimento pessoal e espiritual (Kim et al., 2016), maior valorização da vida, mudança das prioridades da vida (Stanton et al., 2006), e melhores hábitos de saúde e aceitação (Tennen \& Affleck, 2002).

Muitos teóricos (Stagl et al., 2015; Sears et al., 2003; Tedeschi \& Calhoun, 1996) vêm propondo um papel para o ganho percebido no desenvolvimento do crescimento pós-traumático (Post-Traumatic Growth - PTG). Nesses modelos, há diversas variáveis do processo que levam ao ganho perce- bido, tornando-o o resultado. Tedeschi e Calhoun (2015), em seu modelo de PTG, apontam para um crescimento a partir do trauma que resulta em uma reconstrução e reestruturação das crenças globais ou estruturas esquemáticas que guiam os objetivos, a compreensão, a tomada de decisão, e a significação de eventos ameaçadores que acometem os individuos.

Com base no exposto, considera-se que atributos pessoais, como o otimismo, possam afetar a forma como os cuidadores de crianças com câncer percebem ganhos no processo do tratamento dos filhos. O otimismo, na perspectiva deste estudo, é entendido como uma orientação otimista para a vida, ou seja, uma tendência pessoal. Já o ganho percebido, apesar de sugerir uma ideia de resultado de um processo finalizado, trata-se também de uma variável presente durante o processo de enfrentamento.

Com base nos dados trazidos acima, realizou-se um estudo com o objetivo de investigar as relações entre otimismo e ganho percebido em cuidadores de crianças com câncer. O estudo buscou descrever o otimismo em cuidadores de crianças com câncer; investigar o ganho percebido em cuidadores diante da experiência de adoecimento do filho; e analisar as relações entre as variáveis clínicas das crianças e as sociodemográficas dos pais com o otimismo e o ganho percebido.

\section{Método}

\section{Participantes}

Participaram desta pesquisa 60 cuidadores principais de crianças diagnosticadas com câncer, em sua maioria, mulheres (81,7\%), com média de idade igual a 36,5 (DP = 9,17). As crianças estavam em tratamento no Serviço de Onco-Hematologia de um hospital infantil público, vinculado ao Sistema Único de Saúde (SUS), localizado na cidade Vitória, capital do Espírito Santo, ES. Este hospital recebe pacientes advindos de todo o estado do ES, interior e região metropolitana, bem como do leste de Minas Gerais e sul da Bahia, sendo local de referência para o tratamento oncológico infantil. Esses cuidadores constituiram uma amostra de conveniência, considerando os 
seguintes critérios de inclusão: ter um filho e/ ou ser cuidador principal de uma criança com diagnóstico de câncer, que estivesse inscrita no Serviço de Onco-Hematologia do hospital e apresentasse um tempo de diagnóstico mínimo de 1 mês. Foram excluidos os casos de pais cujos filhos se encontravam em situação crítica e/ou internados na Unidade de Terapia Intensiva.

\section{Instrumentos}

Para a caracterização sociodemográfica dos cuidadores e, também, a caracterização das crianças, foram utilizados instrumentos especialmente elaborados, a saber: (a) Questionário de dados sociodemográficos dos cuidadores, para a identificação de dados referentes à composição familiar, moradia, estado civil do cuidador, escolaridade, ocupação, entre outras informações; e (b) Protocolo de registro das caracteristicas da criança, contemplando dados sobre sexo, idade, diagnóstico, tempo de tratamento, gravidade da doença (baixo, médio e alto risco), tipo de tratamento (quimioterapia, radioterapia, cirurgia e tratamentos combinados), entre outros. Os dados dos instrumentos referidos foram obtidos diretamente do cuidador e por meio de consulta aos prontuários médicos das crianças, respectivamente.

O otimismo foi medido por meio do Teste de Orientação da vida (Bandeira et al., 2002), versão em português do Life Orientation Test-LOT, de Scheier et al. (1994), que visa medir o construto de orientação da vida: maneira como as pessoas percebem suas vidas, de uma forma mais otimista ou menos otimista. É constituido por dez itens e dentre esses itens, encontram-se três afirmativas positivas (itens $1,4 \mathrm{e} 10$ ), três afirmativas negativas (itens 3, 7 e g) e quatro questões neutras (2, 5, 6 e 8), que são respondidas em uma escala tipo Likert. O índice geral de otimismo é obtido pela soma da pontuação das questões positivas e negativas, sendo que nas questões negativas a atribuição da pontuação é invertida. Os valores que mais se aproximam de 4 indicam um maior grau de expectativa otimista. O estudo de validação transcultural do instrumento encontrou indicadores satisfatórios relacionados à fidedignidade, validade e consistência interna (valor de $\alpha=0,68$ ) do teste para a população brasileira (Bandeira et al., 2002).

O Inventário de Desenvolvimento Pós-Traumático (Silva et al., 2009) foi utilizado para a medida de ganho percebido. Trata-se da versão em português do Posttraumatic Growth Inventory (PTGI), de Tedeschi e Calhoun (1996), composta de vinte e um itens que expressam mudanças psicológicas positivas ocorridas após um acontecimento adverso. As resposta são fornecidas em uma escala Likert, em que o individuo pode escolher uma opção que varia de o a 5, sendo: 0 = "Eu não experienciei esta mudança como resultado da doença do meu filho"; $1=$ "Eu experienciei muito pouco esta mudança como resultado da doença do meu filho"; 2 = "Eu experienciei pouco esta mudança como resultado da doença do meu filho"; 3 = "Eu não experienciei moderadamente esta mudança como resultado da doença do meu filho"; 4 = "Eu experienciei bastante esta mudança como resultado da doença do meu filho" e 5 = "Eu experienciei completamente esta mudança como resultado da doença do meu filho", de acordo com o seu grau de identificação com o item. O instrumento avalia o ganho percebido considerando quatro dimensões: (a) Percepção de recursos e competências pessoais; (b) Novas possibilidades e valorização da vida; (c) Fortalecimento das relações interpessoais; e (d) Desenvolvimento Espiritual. A tradução apresentou boa consistência interna, sendo, por isso, adequada a sua utilização na população portuguesa (Silva et al., 2009). Na ocasião da realização desta pesquisa, ainda não existia um estudo de validação para a população brasileira, por esta razão utilizou-se a versão portuguesa. O cálculo de consistência interna da versão portuguesa para a amostra de 60 cuidadores de crianças com câncer deste estudo, obteve valores substanciais do Alpha de Cronbach para o escore total $(\alpha=0,79)$ e para os fatores 1 - Percepção de recursos e competências pessoais $(\alpha=0,68)$ e 3 - Fortalecimento das relações interpessoais $(\alpha=0,74)$. Para o fator 2 - Novas possibilidades e valorização da vida ( $\alpha$ $=0,56$ ) e fator 4 - Desenvolvimento espiritual ( $\alpha$ $=0,28$ ), os valores foram moderados e razoáveis, respectivamente. 


\section{Procedimento}

A pesquisa iniciou-se após autorização do Comitê de Ética (Parecer 1.145.387), da Secretaria de Saúde do Estado do Espírito Santo e do Serviço de Onco-Hematologia do Hospital. O recrutamento dos participantes era feito em sala de espera do ambulatório de Onco-Hematologia do hospital e na enfermaria, após consulta diária da pesquisadora à agenda de atendimento do ambulatório e registro de internação da enfermaria e, após a consulta ao prontuário médico da criança para verificar se ela atendia ao critério de inclusão de ter, no minimo, 1 mês de tratamento, os cuidadores eram convidados a participar do estudo. Em seguida, realizava-se uma entrevista para a apresentação da pesquisa e assinatura do Termo de Consentimento Livre e Esclarecido (TCLE), atendendo à resolução ${ }^{\circ}$ 466 de 2012, do Conselho Nacional de Saúde sobre as normas éticas para pesquisa com seres humanos. Somente após essa etapa, a coleta de dados era iniciada, com a aplicação individual dos instrumentos, realizada pela pesquisadora, que é psicóloga. A coleta de dados foi realizada entre outubro e dezembro de 2015. Neste periodo, todos os cuidadores abordados e que atendiam aos critérios de inclusão, aceitaram participar do estudo. Pelo fato de a pesquisadora ser externa ao hospital, foi importante contar com o apoio da equipe, especialmente, para o acesso às fontes de recrutamento dos participantes e viabilização de espaço para a coleta de dados. No ambulatório, a coleta era feita na própria sala de espera do ambulatório. Na enfermaria, a coleta ocorreu próximo ao leito da criança ou corredor do hospital, a depender da escolha do cuidador por não se afastar da criança, mesmo na presença de recreadores na enfermaria. Em ambos os locais, buscou-se criar uma interação favorável junto ao participante, mesmo na ausência de espaço específico para a coleta de dados.

\section{Análise dos dados}

Foi realizada análise descritiva dos dados a partir de medidas como média, mediana, proporção, frequência, amplitude de variação, de acordo com a natureza do dado e com os objetivos da pesquisa. Na análise dos dados obtidos a partir dos instrumentos TOV-R e PTGI, foram consideradas as orientações normativas dos autores. $\mathrm{O}$ teste de correlação de Spearman foi utilizado para identificar a associação entre as variáveis sociodemográficas dos cuidadores e clínicas das crianças, otimismo (Teste de Orientação da Vida - TOV-R) e ganho percebido (Inventário de Desenvolvimento Pós-Traumático). Em seguida, foi realizada uma comparação de médias de grupos independentes para dois niveis de variáveis, utilizando o Teste de Mann-Whitney, com o objetivo de identificar as diferenças significativas entre os resultados de diferentes grupos da amostra (anos de tratamento, quimioterapia). Por fim, foi realizada uma comparação de médias de grupos independentes para dois ou mais niveis de variáveis, por meio do Teste de Kruskal-Wallis, com o objetivo de identificar as diferenças significativas entre os resultados de diferentes grupos (estado civil, diagnóstico) da amostra. O nível de significância utilizado neste estudo foi de 0,05

\section{Resultados}

Com o objetivo de conhecer aspectos da realidade do cuidador, que compõem o contexto de investigação - câncer infantil - foram obtidas características sociodemográficas dos cuidadores e clínicas das crianças com câncer. Os cuidadores eram mulheres $(81,7 \%)$ e homens $(18,3 \%)$, com predominio de grau de parentesco mãe $(80 \%)$ e pai (20\%). A idade dos cuidadores variou entre 20 e 67 (Média = 36,5; DP = 9,17), e um pouco mais da metade deles era casado (55,5\%). A maior parte da amostra se considera religiosa (93,3\%). A escolaridade dos cuidadores variou do ensino fundamental incompleto até o nivel de pós-graduação, com proporção maior de cuidadores com ensino médio completo (33.3\%). Quase metade dos cuidadores (48,3\%) possuia renda inferior a um salário-mínimo.

Sobre as crianças, verificou-se uma distribuição homogênea de meninos e meninas (30 meninos e 30 meninas), com média de idade de 9 anos $(D P=5,28)$. Em relação ao tipo de câncer, verificou-se o predomínio de tumores sólidos (41,7\%), 
seguidos de leucemias (38,3\%) e linfomas ( $9 \%)$. Na ocasião da realização do estudo, a maior parte das crianças estava submetida ao tratamento quimioterápico $(65 \%)$. Além disso, no que concerne ao nivel de gravidade, a maior parte se mostrou de baixo risco (75\%), em seguida médio risco $(18,3 \%)$ e alto risco $(6,7 \%)$. $E$, no que se refere ao tempo de tratamento, a média de meses de tratamento foi de 24,29 (DP = 35,4).

Em relação ao otimismo, verificou-se que os cuidadores de crianças com câncer apresentaram uma média do grau de percepção de vida de 2,64 (DP = 0,57). A partir do cálculo de percentil, os resultados apontaram uma pontuação média localizada no percentil 50 (51,6\%) e 70 (35\%), indicando haver uma percepção otimista da vida. A análise dos itens que determinam o grau de percepção de vida otimista permitiu verificar que "esperar que aconteçam mais coisas boas que coisas ruins", medido pelo item 10, obteve a maior média $(M=3,97)$, seguido de "esperar o melhor em momentos de incerteza" ( $M$ = 3.93), medido pelo item 1 , e de "ser sempre otimista em relação ao futuro" ( $M=3,6)$, medido pelo item 4. A menor média da percepção otimista de vida entre os cuidadores de crianças com câncer foi observada no item 3, que refere o pensamento de "se algo ruim pode acontecer, vai acontecer" $(M=2,1)$.

O ganho percebido, medida pelo PTGl, foi descrita em fatores gerados pelo agrupamento de itens. Desse modo, verificou-se que entre os cuidadores de crianças com câncer, o Fator 4 - Desenvolvimento Espiritual apresentou a maior média ( $M$ = 4.55; $\mathrm{DP}=0,71$ ) e o item deste fator que obteve a maior média foi o item número 15 - Tenho mais compaixão para com os outros ( $M=4,85 ; D P=0,36$ ).

No Fator 1 - Percepção de Recursos e Competências Pessoais ( $M=4,52$; $D P=0,65$ ), 0 item mais referido foi 04 - Sinto que posso contar mais comigo própria, com a média de 4,78 ( $D P=0,71)$; já no Fator 2 - Novas Possibilidades e Valorização da Vida $(M=4,34 ; D P=0,73)$, o item mais mencionado foi 01 - Mudei as minhas prioridades sobre o que é importante na vida $(M=4,5 ; D P=1,14)$. $O$ item que apresentou menor média (21 - Aceito melhor necessitar dos outros, $M=4,43 ; D P=1,29$ ) compõe o Fator 3 - Fortalecimento das Relações Interpessoais ( $M=4,14 ; D P=0,96)$, que obteve a menor média entre os fatores.

No geral, os quatro fatores apresentaram médias elevadas, denotando que os cuidadores de crianças com câncer conseguem perceber ganhos diante da experiência do câncer na criança.

Pelo teste de correlação de Spearman para amostras não paramétricas, houve correlação positiva significativa entre o Otimismo e o Fator 1 - Percepção de Recursos e Competência Pessoais (rho = 0,36; $p=0,004)$, da medida de ganho percebido. Dessa forma, os cuidadores mais otimistas referiram maior ganho percebido, no âmbito de mudanças positivas em seus recursos e competências.

A análise das relações entre as variáveis de interesse indicou correlações significativas entre: estado civil dos cuidadores e ganho percebido; quimioterapia e ganho percebido; e tempo de tratamento, otimismo e ganho percebido. Considerando o estado civil dos cuidadores, houve diferença significativa para o ganho percebido ( $x^{2}(3)$ $=8,315, p=0,40$ ) e o Fator 3 - Fortalecimento das Relações Interpessoais do ganho percebido ( $\chi^{2}(3)$ $=12,751, p=0,005$ ), pelo teste de Kruskal-Wallis. A partir de uma análise dos ranks, verificou-se que os cuidadores casados percebem maior ganho $(33,45)$ e, também, um maior fortalecimento das relações interpessoais $(34,42)$.

Para os grupos de cuidadores, cujas crianças estavam fazendo quimioterapia e para aqueles cujas crianças não estavam submetidas a tal tratamento, houve diferença para a média geral do ganho percebido $(U=214,00 ; p=0,002)$, e para os fatores: Fator 1 - Percepção de Recursos e Competência Pessoais $(U=271,00 ; p=0,022)$, Fator 2 - Novas Possibilidades e Valorização da Vida $(U=249,50 ; p=0,012)$ e Fator 3 - Fortalecimento das Relações Interpessoais $(U=274,50 ; p=0,034)$.

Os dados permitem verificar que os cuidadores das crianças que não estão em quimioterapia percebem mais ganhos durante o processo, como uma maior percepção pessoal de recursos e competências, uma maior abertura a novas possibilidades, valorização da vida e percebem um fortalecimento das suas relações interpessoais. 
O teste de correlação de Spearman demonstrou uma correlação positiva entre o tempo de tratamento e o item 4 - Eu sou sempre otimista com relação ao meu futuro, do Teste de Orientação da Vida (rho = 0,306; $\mathrm{p}=0,018$ ), demonstrando que quanto maior o tempo de tratamento, mais otimistas eles são em relação ao futuro. Considerando o ganho percebido, houve correlação positiva entre o tempo de tratamento e os Fatores 1 - Percepção de Recursos e Competências Pessoais e 2 - Novas Possibilidades e Valorização da Vida (rho = 0,27; $\mathrm{p}=0,03 ;$ rho $=0,36 ; \mathrm{p}=0,004$, respectivamente).

Houve correlação também positiva com os itens 12 - Consigo aceitar o resultado das coisas de forma melhor e 14 - Existem outras oportunidades que não teriam existido antes (rho = 0,25; $\mathrm{p}=0,04 ;$ rho $=0,32 ; \mathrm{P}=0,01$, respectivamente). $\mathrm{A}$ partir destes resultados, verifica-se que quanto maior o tempo de tratamento, mais os cuidadores conseguem compreender seus recursos e competências pessoais e, também, novas possibilidades e uma valorização de suas vidas. Além disso, há a ideia entre eles de ser possivel aceitar o resultado das coisas de uma forma melhor e perceber oportunidades ao longo do tratamento.

Por fim, considerando os grupos com mais e menos de 1 ano de tratamento, houve diferença significativa para o Ganho percebido (Fator 2 - Novas Possibilidades e Valorização da Vida) $(U=265,50$; W = 730,5: $\mathrm{p}=0,006$ ), reforçando o resultado anterior. Analisando as médias de cada grupo, observou-se uma maior percepção de novas possibilidades e valorização da vida $(M=4,60 ; D P=0,52)$.

\section{Discussão}

Adotando um delineamento descritivo, de corte transversal, esta pesquisa analisou as relações entre otimismo e ganho percebido em cuidadores de crianças com câncer. Além disso, foi verificado também se variáveis sociodemográficas dos cuidadores e clínicas das crianças se relacionavam com o otimismo e o ganho percebido.

Em relação ao otimismo, verificou-se que a maior parte dos cuidadores obteve uma pontuação média localizada no percentil 50 (51,6\%) e 70 (35\%), indicando haver uma percepção oti- mista da vida. Quando se considera o percentil $70(35 \%)$, percebe-se que apenas uma parcela dos cuidadores se mostrou mais otimista, porém é preciso considerar as circunstâncias em que esses cuidadores se encontravam quando responderam à pesquisa: em um ambiente hospitalar, com uma criança doente e longe de suas casas. Além disso, uma variedade de fatores deve ser levada em conta quando se fala de otimismo em cuidadores de crianças com câncer: características socioeconômicas, como idade, sexo, nivel de escolaridade (Ellen et al. 2004); fatores intrapsiquicos, como autoestima e autocontrole (Andersson, 1996; Fotiadou et al., 2008) e também fatores da criança, como gravidade dos sintomas e prognóstico. Outros fatores como o suporte social e a tensão do cuidador são potencialmente importantes para o desenvolvimento e manutenção do otimismo (Gardner et al., 2017).

De fato, o otimismo tem sido observado em cuidadores de crianças com câncer (Barrera et al., 2005), inclusive, em um nível mais elevado do que o observado em cuidadores de crianças saudáveis (Fotiadou et al., 2008). De modo mais especifico, cuidadores de crianças com mais tempo de tratamento, apresentaram médias maiores no item da escala de otimismo "Eu sou sempre otimista em relação ao futuro"; indicando possivel influência situacional sobre o otimismo, decorrente do ajustamento psicológico à doença que pode ser observado ao longo do tempo. Em outros contextos, como relatam Bozkurt et al. (2019), em pesquisa realizada na Turquia, não houve correlação significativa entre o tempo de diagnóstico e o otimismo; de maneira contrária ao encontrado neste estudo, as análises dos autores mostraram que quanto maior o tempo após o diagnóstico, menores os níveis de otimismo. Ainda são escassos os estudos que investigam o otimismo em cuidadores de crianças com câncer, havendo limitação nas inferências a respeito do entendimento do otimismo enquanto relacionado a traços de personalidade ou a determinantes situacionais relativos à doença. O que podemos inferir é que o contexto dos cuidadores de crianças com câncer influencia na percepção otimista de 
vida. No estudo de Fayed et al. (2011), os modelos de análise indicaram que o otimismo foi menos influenciado por características da doença, como o tipo de câncer e o tempo do diagnóstico; e mais influenciados por traços de personalidade de pais. Particularmente, no presente estudo não foram investigadas características intrapsiquicas dos cuidadores que pudessem estar relacionadas ao otimismo, constituindo, portanto, uma limitação. Em consonância com o estudo de Fayed et al. (2011), sugere-se que novas pesquisas incluam a medida do otimismo ao longo do tempo, a fim de verificar possiveis mudanças do fenômeno no contexto do câncer infantil.

Um dos objetivos desse estudo foi investigar o ganho percebido em cuidadores de crianças com câncer. A esse respeito, é válido retomar neste ponto da discussão o conceito desse fenômeno, dada a sua recente inserção na literatura nacional sobre o câncer infantil. O ganho percebido trata de uma mudança positiva observada pelo individuo que passa por uma adversidade, podendo ser referido também como crescimento pós-traumático (Shaw et al., 2005). Trata-se do processo de observar benefícios na adversidade, diminuindo assim as implicações negativas do mesmo (Seligman \& Csikszentmihalyi, 2014).

Nesse estudo, os cuidadores das crianças que estavam em tratamento de câncer referiram perceber ganhos, por meio da experiência de ter um filho com câncer. Particularmente, o ganho percebido apresentou maior média no Fator 4 - Desenvolvimento Espiritual, o qual abrigava, também, o item mais referido: "Tenho mais compaixão para com os outros". Em revisão de literatura sobre coping espiritual, ajustamento psicossocial e saúde mental de jovens com doenças crônicas, Reynolds et al. (2016) concluíram que a espiritualidade e a religião contribuem de modo significativo para o ajustamento psicossocial dos individuos diante da adversidade representada pelo câncer e seu tratamento. Nessa mesma direção, Vitorino et al. (2018) referiram que a religião e a fé são um importante recurso de enfrentamento para mães de crianças com câncer. Além disso, afirmam que cuidadores que se adaptam melhor à situação, em geral, apresentam maior grau de estratégias de enfrentamento espirituais/religiosas positivas. Esse estudo mostrou que, quando se trata de cuidadores de crianças com câncer, a religiosidade também se constitui caminho de crescimento em meio à adversidade. Passar pela experiência de ter um filho com câncer pode representar ganhos, especialmente, relacionados ao desenvolvimento espiritual. O fato destes cuidadores referirem mais compaixão, pode demonstrar um aumento na possibilidade de maior empatia, altruísmo e solidariedade com as pessoas (Silva et al., 2009; Kim et al., 2007).

Para a melhor compreensão do ganho percebido, foram investigadas suas relações com variáveis sociodemográficas dos cuidadores (estado civil) e clínicas das crianças (quimioterapia e tempo de tratamento); as associações significativas são discutidas, a seguir.

Os cuidadores casados perceberam maior ganho durante o tratamento do câncer da criança e, também, um maior fortalecimento das relações interpessoais (Fator 3). Em contrapartida aos estudos que se referem à deteriorização das relações conjugais durante a experiência de ter um filho com câncer (Dahlquist et al., 1996), os achados desse estudo indicam outra direção, a saber: o fortalecimento das relações interpessoais, particularmente, entre os cuidadores casados. Em consonância com esse achado, Wiener et al. (2017) verificaram que os efeitos do câncer infantil no casamento e na qualidade de vida dos pais não são, necessariamente, debilitantes. Em seu estudo, esses autores verificaram que, apesar de muitos casais encontrarem dificuldades ao longo do tratamento do filho, o fato de conseguirem manter uma boa comunicação e encontrarem formas de lidar com o estresse pode aumentar o nivel de satisfação conjugal (Wiener et al., 2017). Em âmbito nacional, o estudo de Quintana (2017) é consistente com esses dados, uma vez que ao investigar a reestruturação familiar diante do câncer, relata a ocorrência de mudanças nos vínculos, no sentido de um fortalecimento emocional, de modo que a doença pode ser um evento de união e reconciliação familiar. 
É importante ressaltar que o impacto de uma doença grave, como o câncer, na relação conjugal dos pais deve ser analisado juntamente com outras variáveis que podem alterar a percepção do estressor e, em consequência, seus efeitos sobre o funcionamento familiar e o ganho percebido. Norberg et al. (2005) alertaram para a realidade da família da criança com câncer, a qual é caracterizada por um acúmulo de estressores, que diferem em duração, previsibilidade e impacto. Van Schoors et al. (2017), em revisão de literatura, apresentam as mudanças nos relacionamentos familiares, trazendo maior clareza quanto aos aspectos positivos e negativos dessas mudanças. No lado positivo, observou-se melhor percepção dos traços pessoais e comportamentais do parceiro, maior capacidade de resolver conflitos, maior confiança e flexibilidade para com o parceiro e maior apoio; e, no lado negativo, esses autores encontraram piora na relação íntima e sexual do casal, pois esses acabam investindo muito da sua energia física e emocional para lidar com a doença do filho, dando pouca ou nenhuma atenção à intimidade e às relações sexuais.

Nesse ponto da discussão, entende-se que a experiência do câncer pode trazer mudanças positivas e negativas para o relacionamento conjugal. Nesse estudo, a associação entre estar casado e ter maior percepção de ganhos no contexto do câncer infantil parece indicar um impacto positivo, com o fortalecimento dos relacionamentos. Mais uma vez, mostra-se fundamental que a assistência à criança com câncer inclua também a familia. Essa assistência, por sua vez, deve partir de uma avaliação de risco psicossocial familiar ao longo do processo de tratamento (Alderfer et al., 2009), sendo assim possivel adequar as estratégias de intervenção que possivelmente contribuirão para o desenvolvimento da competência dos cuidadores e de seu papel como fonte de suporte social reciproco, aumentando as chances de passar pela experiência do câncer do filho percebendo benefícios na adversidade.

Sobre as características clínicas das crianças com câncer, análises inferenciais mostraram resultados significativos. Os cuidadores das crianças que não estavam em quimioterapia referiram maior ganho percebido, maior percepção de recursos e competências pessoais (Fator 1), maior abertura de novas possibilidades e valorização da vida (Fator 2) e, também, maior fortalecimento das relações interpessoais (Fator 3). Nesse estudo, as situações em que a criança não estava em quimioterapia eram justificadas pelo fato de que essa modalidade do tratamento já havia sido concluida ou por não ter sido parte do protocolo de tratamento da doença. No caso de cuidadores de crianças que já haviam finalizado a quimioterapia, essa condição indicava uma fase do tratamento em que várias etapas já tinham sido superadas, como o diagnóstico, o início do tratamento quimioterápico e seus efeitos colaterais, contribuindo para uma percepção do câncer como um evento passivel de ser controlado.

Outra variável clínica da criança que se associou ao ganho percebido foi o tempo de tratamento. Mais especificamente, o tempo de tratamento em meses se associou ao ganho percebido, por meio das correlações apresentadas com os itens: "Consigo aceitar o resultado das coisas de forma melhor"; "Existem outras oportunidades que não teriam existido antes"; e com os fatores "Percepção de recursos e competências pessoais" e "Novas possibilidades e valorização da vida". Em outra análise, que agrupou os cuidadores considerando o tempo de tratamento, verificou-se que os cuidadores das crianças com mais de um ano de tratamento também reportaram maior ganho percebido, pela correlação com o Fator 2 - Novas possibilidades e valorização da vida. Estudos que investigaram relações entre o tempo de tratamento e a variável ganho percebido encontraram resultados divergentes. Se, de um lado, Phipps et al. (2007) não encontraram associação entre essas variáveis; outros estudos confirmaram a associação entre o tempo de tratamento e o ganho percebido, principalmente aqueles sobre crescimento pós-traumático com cuidadores de crianças com câncer (Barakat et al., 2006; Michel et al., 2010) e, também, com os próprios pacientes, tanto adolescentes, quanto adultos (Barakat et al., 2006, Cordova et al., 2017. 
Sears et al., 2003). Especificamente no estudo de Barakat et al. (2006), as familias conseguiam identificar muitas mudanças positivas durante o tratamento e, também, ao final do mesmo, com percepções de crescimento relativo ao que eles pensam sobre suas vidas, seus planos para o futuro e o quão cuidadores eles se tornaram.

Por fim, o otimismo apresentou uma correlação positiva com o ganho percebido, particularmente, no fator Percepção de Recursos e Competência Pessoais. Nesse sentido, o otimismo, em geral, é visto como um recurso pessoal que pode auxiliar no desenvolvimento do ganho percebido, pois ele pode favorecer a visualização de ganhos mediante a experiências negativas. Corroborando com essa ideia, Llewellyn et al. (2013), em estudo sobre preditores do ganho percebido, identificou nos resultados que tanto o otimismo, quanto as estratégias adaptativas, se relacionavam com o ganho percebido e, além disso, os autores também apontam estas três variáveis como preditores de resiliência.

O estudo realizado encontrou limitações metodológicas referentes ao recrutamento da amostra, uma vez que o estudo foi autorizado para a coleta de dados nos espaços do ambulatório e internação hospitalar. Desse modo, foram excluídos cuidadores cujos filhos estavam internados em UTI. O mesmo ocorreu para os casos em que a criança estava em estado grave. Neste caso, aspectos éticos e de cuidado ao paciente e à família conduziram a tomada de decisão pela exclusão desses casos, evitando a exposição do participante a questões que pudessem gerar ainda mais sofrimento psicológico; de modo mais específico, falar de aspectos positivos da experiência do câncer quando a condição de gravidade se fazia presente de forma aguda. Barreto e Boeckel (2019) ressaltam que o ambiente hospitalar, assim como UTIs são locais geradores de estresse e com uma grande carga emocional para os pacientes e familiares. Essa è uma das razões pelas quais a investigação nesses contextos, especialmente, quando o pesquisador não compõe a equipe de saúde, torna-se um desafio. No caso desse estudo, assumiu-se a limitação metodológica representada pela tomada de decisão referida. Essa limitação pode ser superada em estudos posteriores, com foco na investigação de variáveis relacionadas ao crescimento pós-traumático e outros indicadores de saúde mental positiva, em cuidadores de crianças e adolescentes que estejam em condição de maior gravidade. Estudos nessa direção devem ser delineados de modo a considerar a necessidade de amenizar os riscos da abordagem à familia nesses contextos, o que inclui desde a organização de um espaço físico adequado para a coleta de dados e estratégias de suporte psicológico.

Outras limitações metodológicas foram observadas, como a ausência de estudo de validação do PTGI para a população brasileira, sendo necessário a utilização da versão para a população portuguesa. Para amenizar essa limitação, procedeu-se ao cálculo de consistência interna do PTGI na amostra do estudo. Os achados a esse respeito se constituem em contribuições do estudo, uma vez que foram encontrados indicadores satisfatórios para os fatores 1, 2 e 3. Em relação ao fator 4, a consistência interna se mostrou razoável, o que pode ser explicado pelo número reduzido da amostra. Estudo posterior confirmou a estrutura fatorial da escala em uma população brasileira, bem como indicadores satisfatórios de validade, com coeficiente alfa variando entre 0,72 e 0,92 (Campos \& Trentini, 2019).

É relevante destacar que o estudo se insere na área da Psicologia da Saúde, mas o foco em aspectos positivos da experiência de adversidade, representada pelo câncer na criança, revela uma interface com a Psicologia Positiva. Essa interface pode ser promissora, subsidiando intervenções que integrem as forças e as fragilidades que a familia traz para o enfrentamento do adoecimento da criança. De acordo com Calvetti et al. (2007), essa aproximação de áreas é uma tendência, e poderá auxiliar na compreensão dos aspectos envolvidos no enfrentamento da doença bem como na manutenção da saúde da pessoa.

Este estudo buscou analisar e compreender as relações entre otimismo e ganho percebido em cuidadores de crianças com câncer. De modo geral, os cuidadores se mostraram otimistas, mesmo considerando o contexto em que estavam inseridos, o tratamento do câncer de seu filho. 
Os resultados mostram também a importância de se pensar em intervenções para estes cuidadores e/ou pais de crianças com câncer. As intervenções devem ser pensadas de maneiras distintas conforme este estudo discute, levando em consideração aspectos como tempo e fase do tratamento. Além disso, promover o suporte social, que se mostrou importante para a percepção de ganho, favorecer a adaptação psicológica diante da adversidade, valorizando características individuais - como o otimismo - que possam aumentar as chances dos pais ressignificarem a sua experiência e perceberem ganhos advindos dessa.

\section{Referências}

Alderfer, M. A., Mougianis, I., Barakat, L. P., Beele, D., DiTaranto, S., Hwang, W.-T., Reilly, A.T. \& Kazak, A. E. (2009). Family psychosocial risk, distress, and service utilization in pediatric cancer. Cancer, 115(S18), 43394349. https://doi.org/10.1002/cncr.24587

Andersson G. (1996). The benefits of optimism: a metaanalytic review of the life orientation test. Pers Individ Differences, 21(5), 719-725. https://doi.org/10.1016/01918869(96)00118-3

Andrea, M. L. M. (2008). Oncologia pediátrica. In V. A. Carvalho et al. (Orgs.), Temas em psico-oncologia (pp. 477-495). Summus.

Barakat, L. P., Alderfer, M. A., \& Kazak, A. E. (2006). Posttraumatic growth in adolescent survivors of cancer and their mothers and fathers. Journal of Pediatric Psychology, 31(4), 413-419. https://doi.org/10.1093/ipepsy/jsj058

Barrera M., D'Agostino N., Gammon J., Spencer L., \& Baruchel S. (2005). Health-related quality of life and enrollment in phase 1 trials in children with incurable cancer. Palliat Support Care, 3, 191-196. https://doi. org/10.1017/S1478951505050315

Barreto, J. B. V., \& Boeckel, M. G. (2019). Regulação emocional e conjugalidade: vivência de pais com filhos internados em Unidade de Terapia Intensiva. Contextos Clínicos, 12(1), 342-372. http://dx.doi.org/10.4013/ctc.2019.121.14

Bozkurt, G., Inal, S., Yantiri, L., \& Alparslan, Ö. (2019). Relationship between coping strategies, religious attitude, and optimism of mothers of children with cancer. Journal of Transcultural Nursing, 30(4), 365-370. https:// doi.org/10.1177/1043659618818714

Calvetti, P. U., Muller, M. C., \& Nunes, M. L. T. (2007). Psicologia da saúde e psicologia positiva: perspectivas e desafios. Psicologia: Ciência e Profissão, 27(4), 706-717. https://dx.doi.org/10.1590/S1414-98932007000400011

Campos, J. O. C., \& Trentini, C. M. (2019). Análise Fatorial Confirmatória da Versão Brasileira do Inventário de Crescimento Pós-Traumático. Avaliação Psicológica, 18(1), 50-57. https://dx.doi.org/10.15689/ ap.2019.1801.14667.06
Compas, B. E., Desjardins, L., Vannatta, K., Young-Saleme, T., Rodriguez, E. M., Dunn, M., Bemis, H., Snyder, S., \& Gerhardt, C. A. (2014). Children and adolescents coping with cancer: Self-and parent reports of coping and anxiety/depression. Health Psychology, 33(8), 853. https://doi.org/10.1037/hea0000083

Cordova, M. J., Riba, M. B., \& Spiegel, D. (2017). Post-traumatic stress disorder and cancer. The Lancet Psychiatry, 4(4), 330-338. https://doi.org/10.1016/S22150366(17)30014-7

Dahlquist, L. M., Czyzewski, D. I., \& Jones, C. L. (1996). Parents of children with cancer: A longitudinal study of emotional distress, coping style, and marital adjustment two and twenty months after diagnosis. Journal of pediatric psychology, 21(4), 541-554. https:// doi.org/10.1093/jpepsy/21.4.541

da Silva, B. C. D. A., dos Santos, M. A., \& de Oliveira Cardoso, É. A. (2019). Vivências de familiares de pacientes com câncer: revisitando a literatura. Revista da SPAGESP, 20(1), 140-153

de Siqueira, H. C. H., Bick, M. A., Sampaio, A. D., de Medeiros, A. C., de Siqueira Bento, A., \& Severo, D. F. (2019). Repercussões do câncer infantil no ambiente familiar. Revista Renome, 8(1), 20-29.

Ellen E. K., Remes J., Sovia U. (2004). Social and developmental predictors of optimism from infancy to early adulthood. Social Indicators Research, 69, 219-242. https://doi.org/10.1023/B:SOCl.0000033591.80716.07

Fayed, N., Klassen, A. F., Dix, D., Klaassen, R., \& Sung, L. (2011). Exploring predictors of optimism among parents of children with cancer. Psycho-Oncology, 20(4), 411-418. https://doi.org/10.1002/pon.1743

Fotiadou, M., Barlow, J. H., Powell, L. A., \& Langton, H. (2008). Optimism and psychological well-being among parents of children with cancer: an exploratory study. Psycho-Oncology, 17(4), 401-409. https://doi. org/10.1002/pon.1257

Gardner, M. H., Mrug, S., Schwebel, D. C., Phipps, S., WheLan, K., \& Madan-Swain, A. (2017). Demographic, medical, and psychosocial predictors of benefit finding among caregivers of childhood cancer survivors. Psycho-Oncology, 26(1), 125-132. https://doi.org/10.1002/pon.4014

Gomes I. P., \& Collet N. (2010). Distressful symptoms related to chemotherapy from the perspective of children: a qualitative research. Online Brazilian Journal of Nursing. 9(2). https://doi.org/10.5935/1676-4285.20103045

Harding, S., Sanipour, F., \& Moss, T. (2014). Existence of benefit finding and posttraumatic growth in people treated for head and neck cancer: a systematic review. PeerJ, 2, e256. https://doi.org/10.7717/peerj.256

Hildenbrand, A. K., Alderfer, M. A., Deatrick, J. A., \& Marsac, M. L. (2014). A mixed methods assessment of coping with pediatric cancer. Journal of psychosocial oncology, 32(1), 37-58. https://doi.org/10.1080/07347 332.2013 .855960

Instituto Nacional de Câncer José Alencar Gomes da Silva (2019). Tratamento do câncer. INCA. https://WwW. inca.gov.br/tratamento 
Kazak, A. E., \& Baxt, C. (2007). Families of infants and young children with cancer: a post-traumatic stress framework. Pediatric Blood Cancer, 49(7), 1109-1113. https://doi.org/10.1002/pbc.21345

Kim, Y., Schulz, R., \& Carver, C. S. (2007). Benefit finding in the cancer caregiving experience. Psychosomatic Medicine, 69(3), 283-291. https://doi.org/10.1097/ PSY.ob013e $3180417 \mathrm{cf} 4$

Kim, J., Kim, M., \& Park, S. H. (2016). Exploring the relationship among posttraumatic growth, life satisfaction, and happiness among Korean individuals with physical disabilities. Psychological reports, 119(1), 312-327. https:// doi.org/10.1177/0033294116653954

Kohlsdorf, M., \& Costa Junior, A. L. (2012). Impacto psicossocial do câncer pediátrico para pais: revisão da literatura. Paidéia (Ribeirão Preto), 22(51), 119-129. https://doi.org/10.1590/S0103-863X2012000100014

Llewellyn, C. D., Horney, D. J., McGurk, M., Weinman, J., Herold, J., Altman, K., \& Smith, H. E. (2013). Assessing the psychological predictors of benefit finding in patients with head and neck cancer. Psycho-Oncology,22(1), 97-105. https://doi.org/10.1002/pon.2065

Mavandadi, S., Dobkin, R., Mamikonyan, E., Sayers, S., Ten Have, T., \& Weintraub, D. (2014). Benefit finding and relationship quality in Parkinson's disease: A pilot dyadic analysis of husbands and wives. Journal of Family Psychology, 28(5), 728. https://doi.org/10.1037/a0037847

Michel, G., Taylor, N., Absolom, K., \& Eiser, C. (2010). Benefit finding in survivors of childhood cancer and their parents: Further empirical support for the Benefit Finding Scale for Children. Child: Care, health and development, 36(1), 123-129. https://doi.org/10.1111/j. 1365-2214.2009.01034.X

Ministério da Saúde. (2020). Instituto Nacional de Câncer José Alencar Gomes da Silva. Estimativa 2020: Incidência de Câncer no Brasil. Rio de Janeiro.

Norberg, A. L., Lindblad, F., Boman, K. K. (2005). Coping strategies in parents of children with cancer. Social, Science \& Medicine, 60, 965-975. https://doi. org/10.1016/j.socscimed.2004.06.030

Phipps, S. (2007). Adaptative style in children with cancer: Implications for a positive psychology approach. Journal of Pediatric Psychology, 32(9), 1055-1066. https:// doi.org/10.1093/jpepsy/jsmo60

Phipps, S., Long, A. M. \& Ogden, J. (2007). Benefit finding scale for children: preliminary findings from a childhood cancer population. Journal of Pediatric Psychology, 32 , 1264-1271. https://doi.org/10.1093/jpepsy/jsl052

Quintana, A. M. et al. (2017, nov.). Lutos e lutas: Reestruturações familiares diante do câncer em uma criança/ adolescente. Psicologia Argumento, 29(65), 145-164.

Reynolds, N., Mrug, S., Wolfe, K., Schwebel, D., \& Wallander, J. (2016). Spiritual coping, psychosocial adjustment, and physical health in youth with chronic illness: a meta-analytic review. Health psychology review, 10(2), 226-243. https://doi.org/10.1080/17437199.2016.1159142
Salvador, Á., Crespo, C., Martins, A. R., Santos, S., \& Canavarro, M. C. (2015). Parents' perceptions about their child's illness in pediatric cancer: Links with caregiving burden and quality of life. Journal of child and family studies, 24(4), 1129-1140. https://doi.org/10.1007/ s10826-014-9921-8

Scheier, M. F., \& Carver, C. S. (1985). Optimism, coping, and health: assessment and implications of generalized outcome expectancies. Health Psychology, 4, 219-247. https://doi.org/10.1037/0278-6133.4.3.219

Sears, S. R., Stanton, A. L., \& Danoff Burg, S. (2003). The yellow brick road and the emerald city: benefit finding, positive reappraisal coping, and posttraumatic growth in women with early stage breast cancer. Health Psychology, 22(5), 487-97. https://doi.org/10.1037/0278-6133.22.5.487

Segerstrom, S. C., Carver, C. S., \& Scheier, M. F. (2017). Optimism. In Robinson, M. D., \& Eid, M. (Eds.), The happy mind: Cognitive contributions to well-being (pp. 195-212). Springer International Publishing.

Seligman, M. E., \& Csikszentmihalyi, M. (2014). Positive psychology: An introduction. In Csikszentmihalyi, M., \& Larson, R., Flow and the foundations of positive psychology (pp. 279-298). Springer. https://doi.org/10.1007/97894-017-9088-8_18

Silva, S. I. M., Moreira, H. I. C. Pinto, S. M. A., \& Canavarro, M. C. C. S. P. (2009). Cancro da mama e desenvolvimento pessoal e relacional: estudo das características psicométricas do Inventário de Desenvolvimento Pós-Traumático (Psttraumatic Growth Inventory) numa amostra de mulheres da população portuguesa. RIDEP, 28(2), 105-133.

Stagl, J. M., Antoni, M. H., Lechner, S. C., Bouchard, L. C., Blomberg, B. B., Glück, S., Derhagopian, R. P., \& Carver, C. S. (2015). Randomized controlled trial of cognitive behavioral stress management in breast cancer: A brief report of effects on 5-year depressive symptoms. Health psychology, 34(2), 176-180. https:// doi.org/10.1037/hea0000125

Stanton A. L., Bower, J. E., \& Low, C. A. (2006). Posstraumatic growth after cancer. In L. G. Calhoun, \& R. G. Tedeschi (Eds.), Handbook of posstraumatic growth (pp. 138-75). Lawrence Erlbaum Associates.

Taylor, S. E. (1983). Adjustment to threatening events: A theory of cognitive adaptation. American Psychologist, 38(11), 1161-1173. https://doi.org/10.1037/ 0003-066X.38.11.1161

Tedeschi, R. G., \& Calhoun, L. G. (1996). The Posttraumatic Growth Inventory: Measuring the positive legacy of Trauma. Journal of Traumatic Stress, 9, 455-471. https:// doi.org/10.1002/jts.2490090305

Tedeschi, R. G., Calhoun, L. G., \& Groleau, J. M. (2015) Clinical applications of posttraumatic growth. Positive psychology in practice: Promoting human flourishing in work, health, education and everyday life, 2, 503-518. https://doi.org/10.1002/9781118996874.ch30

Tennen, H., \& Affleck, G. (2002). Benefit-finding and benefit-reminding. In C. R. Snyder, \& S. J. Lopez (Eds.), Handbook of positive psychology (pp. 584-597). Oxford University Press. 
Van Schoors, M., Caes, L., Alderfer, M. A., Goubert, L., \& Verhofstadt, L. (2017). Couple functioning after pediatric cancer diagnosis: a systematic review. Psycho-Oncology, 26(5), 608-616. https://doi.org/10.1002/pon.4204

Vitorino, L. M., Lopes-Júnior, L. C., de Oliveira, G. H., Tenaglia, M., Brunheroto, A., Cortez, P. J. O., \& Lucchetti, G. (2018). Spiritual and religious coping and depression among family caregivers of pediatric cancer patients in Latin America. Psycho-Oncology, 27(8), 1900-1907. https://doi.org/10.1002/pon.4739

Wiener, L., Battles, H., Zadeh, S., Pelletier, W., Arruda-Colli, M. N. F., \& Muriel, A. C. (2017). The perceived influence of childhood cancer on the parents' relationship. Psycho-oncology, 26(12), 2109-2117. https:// doi.org/10.1002/pon.4313

Wiener, L., Kazak, A. E., Noll, R. B., Patenaude, A. F., \& Kupst, M. J. (2015). Standards for the psychosocial care of children with cancer and their families: an introduction to the special issue. Pediatric blood \& cancer, 62(S5), S419-S424. https://doi.org/10.1002/pbc.25675

Willard, V. W., Hostetter, S. A., Hutchinson, K. C., Bonner, M. J., \& Hardy, K. K. (2016). Benefit finding in maternal caregivers of pediatric cancer survivors: a mixed methods approach. Journal of Pediatric Oncology Nursing, 33(5), 353-360. https://doi.org/10.1177/1043454215620119

Williams, N. A., Davis, G., Hancock, M., \& Phipps, S. (2010). Optimism and pessimism in children with cancer and healthy children: confirmatory factor analysis of the youth life orientation test and relations with health-related quality of life. Journal of pediatric psychology, 35(6), 672-682. https://doi.org/10.1093/jpepsy/jspo84

Yi, J., Zebrack, B., Kim, M. A., \& Cousino, M. (2015). Posttraumatic growth outcomes and their correlates among young adult survivors of childhood cancer. Journal of pediatric psychology, 40(9), 981-991. https:// doi.org/10.1093/jpepsy/jsv075

\section{Larissa Bessert Pagung}

Mestre em Psicologia pela Universidade Federal do Espirito Santo (UFES), em Vitória, ES, Brasil; psicóloga da AFECC Hospital Santa Rita de Cássia, em Vitória, ES, Brasil; professora da Faculdade Multivix Cariacica, em Cariacica, ES, Brasil.

\section{Kelly Ambrósio Silveira}

Doutora em Psicologia pela Universidade Federal do Espírito Santo (UFES), em Vitória, ES, Brasil.

\section{Alessandra Brunoro Motta}

Doutora em Psicologia pela Universidade Federal do Espirito Santo (UFES), em Vitória, ES, Brasil; professora da Universidade Federal do Espírito Santo (UFES), em Vitória, ES, Brasil.

\section{Endereço para correspondência}

Larissa Bessert Pagung

Universidade Federal do Espírito Santo

Av. Fernando Ferrari, 514. Prédio Professor Lídio de Souza

Goiabeiras, 29075-910

Vitória, ES, Brasil

Os textos deste artigo foram revisados pela Poá Comunicação e submetidos para validação do(s) autor(es) antes da publicação. 